\title{
Comparison of spermiograms of infertile men before and during the COVID-19 pandemic
}

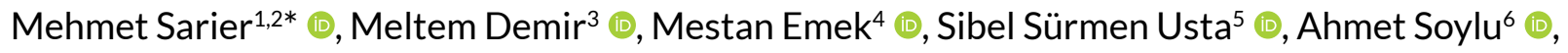 \\ Elçin Yenidünya Konuk ${ }^{7}$ (1), Hasan Turgut ${ }^{8}$ (1)
}

\section{SUMMARY}

OBJECTIVE: Since the start of the COVID-19 pandemic, there has been interest in the impact of both SARS-CoV-2 infection and pandemic-induced social restrictions on male reproductive health. This study aimed to evaluate the spermiogram values of men who presented for infertility during the pandemic compared with the previous 2 years.

METHODS: Patients who presented to a urology outpatient clinic for the first time due to infertility were included. The patients' age, semen volume, and spermiogram results were recorded. Based on the presentation date, the patients were divided into prepandemic group 1 (March 2018-February 2019), prepandemic group 2 (March 2019-February 2020), and pandemic group (March 2020-February 2021) for comparison.

RESULTS: A total of 594 patients were included. There was no significant difference between the three groups in terms of the number of patients who presented for infertility $(207,190$, and 197 patients, respectively; $p=0.691)$. The mean age was $36.6 \pm 7.2$ in the prepandemic group 1 , $35.5 \pm 7.1$ in the prepandemic group 2, and 33.1 \pm 6.3 in the pandemic group. Patients who presented during the pandemic were significantly younger ( $<<0.001)$. There were no differences between the groups in terms of semen volume ( $p=0.910$ ) or rates of normospermia and pathological spermiogram findings ( $p=0.222$ ). CONCLUSIONS: In the first year of the COVID-19 pandemic, there was no significant difference in the number of patients who presented for infertility or in their spermiogram results compared with 2018 and 2019. However, it is noteworthy that the patients were significantly younger during the pandemic than in the previous 2 years.

KEYWORDS: COVID-19 pandemic. Male infertility. Spermiogram. SARS-CoV-2. Semen analysis.

\section{INTRODUCTION}

The novel coronavirus disease (named COVID-19 by the World Health Organization [WHO]) has spread from a cluster of unexplained pneumonia cases in Wuhan, China, to a global public health emergency, affecting every country in the world within months, and it was declared a pandemic by the WHO on March 11, 20201. Throughout the intervening year, research has been conducted to understand the health consequences of both SARS-CoV-2 infection and pandemic-induced social restrictions. Before the COVID-19 pandemic, infertility was estimated to affect between 8 and $12 \%$ of couples of reproductive age worldwide, with male infertility solely responsible for $20-30 \%$ of these cases and a contributing factor in $50 \%$ of all cases $^{2-4}$. Since the start of the COVID-19 pandemic, several studies have been conducted on the effect of the SARS-CoV-2 virus on the male reproductive system, especially testicular functions. These studies mostly focused on histopathological changes in testicular and semen parameters caused by COVID-19 infection, the presence of virus in reproductive organs and semen, and its effects on sex hormones ${ }^{5-8}$. However, sexual and reproductive health is not simply an absence of disease or dysfunction but a state of physical, mental, and social well-being related to every facet of reproduction and sexuality ${ }^{9}$. Despite the vaccination

\footnotetext{
${ }^{1}$ Istinye University, Department of Urology - Istanbul, Turkey.

${ }^{2}$ Medical Park Hospital, Department of Urology - Antalya, Turkey.

${ }^{3}$ Antalya Bilim University, Faculty of Health Science - Antalya, Turkey.

${ }^{4}$ Akdeniz University, Department of Public Health - Antalya, Turkey.

${ }^{5}$ Medical Park Hospital, Department of Obstetrics and Gynaecology - Antalya, Turkey.

${ }^{6}$ Atlas University, Department of Urology - Istanbul, Turkey.

${ }^{7}$ Provincial Directorate of Health, Public Health Laboratory - Antalya, Turkey.

${ }^{8}$ Avrasya University, Faculty of Health Science - Trabzon, Turkey.

*Corresponding author: drsarier@gmail.com

Conflicts of interest: the authors declare there is no conflicts of interest. Funding: none.

Received on November 20, 2021. Accepted on November 21, 2021.
} 
programs are currently underway, a concern that the pandemic will continue in the long term raises the question of what impact the pandemic-imposed lifestyle changes will have on male reproductive health. Considering the notable changes in sexual behavior observed in society since the start of the COVID-19 pandemic ${ }^{10,11}$, community-based studies of male reproductive health during the pandemic are also needed. The results from these studies will be important in further elucidating the social effects of COVID-19.

The aim of this study was to investigate semen parameters in men who presented to the urology clinic due to infertility in the first year of the pandemic and to compare the results with those in the previous 2 years.

\section{METHODS}

This study included men who presented to the urology outpatient clinic of the Medical Park Antalya Hospital Complex between March 2018 and February 2021 due to infertility. Inclusion criteria were as follows: the patients who failed to achieve pregnancy for more than 12 months despite regular unprotected sexual intercourse ${ }^{4}$ and those who presented to our clinic with this complaint for the first time. Patients who presented for follow-up and those who had previous infertility treatment were excluded from this study.

After obtaining a detailed history and performing thorough physical examination, semen samples were collected in sterile tubes by masturbation after an average of 3-5 days of sexual abstinence and were analyzed after liquefaction at $37^{\circ} \mathrm{C}$ for $30 \mathrm{~min}$. A $5 \mu \mathrm{L}$ aliquot of semen was loaded into a Makler counting chamber to determine basic spermatozoa concentration and percentage of motile spermatozoa according to WHO 2010 criteria, and morphological evaluation was performed using light microscopy according to Kruger criteria after staining ${ }^{12}$. Sperm concentration was expressed as sperm per milliliter of semen; motility and morphology were expressed as percentages. The results were evaluated using the following WHO criteria for lower reference limits: semen volume $(\mathrm{mL}): 1.5 \mathrm{~mL}$; sperm concentration (sperm/mL): 15 million/mL; total motility: $40 \%$; progressive motility: $32 \%$, vitality: $58 \%$, and morphology (normal forms): $4 \%{ }^{12}$. All semen samples were evaluated by the same specialist. Patients' age, semen volume, and spermiogram results were recorded. Based on the presentation date, the patients were divided into prepandemic group 1 (March 2018-February 2019), prepandemic group 2 (March 2019-February 2020), and pandemic group (March 2020-February 2021). The results were compared between these groups.

\section{Ethical considerations and statistical analysis}

This retrospective study was approved by the Antalya Medical Park Hospital Complex Ethics Committee (approval no: 2021/03) and was carried out in accordance with the Declaration of Helsinki (1975). All statistical analyses were performed using SPSS statistical software (SPSS for Windows version 18.0; SPSS Inc., Chicago, IL, USA). Age and semen volume were expressed as mean \pm standard deviation. The normality of the data distribution was assessed using the Kolmogorov-Smirnov test. Age and semen volume were compared using the Kruskal-Wallis test, and the Mann-Whitney $U$ test with the Bonferroni correction was used for pairwise comparisons. Relative differences in the distribution of normospermia and pathological spermiogram rates by year were compared with the chi-square test. P-values $<0.05$ were considered statistically significant.

\section{RESULTS}

A total of 594 patients were included in the study. The number of patients who presented to the urology clinic for infertility did not differ significantly by year (in chronological order: 207 , 190, and 197; $p=0.691$ ). The mean age was $36.6 \pm 7.2$ in the prepandemic group 1, 35.5 \pm 7.1 in the prepandemic group 2, and $33.1 \pm 6.3$ in the pandemic group. Patients who presented during the pandemic were significantly younger $(p<0.001)$. There was no difference in semen volume between the three groups $(\mathrm{p}=0.910)$. The analysis of spermiogram results revealed no significant differences in normospermia and pathological spermiogram rates by year $(\mathrm{p}=0.222)$. The spermiogram results of all three groups are shown in Table 1.

\section{DISCUSSION}

To the best of our knowledge, this study is the first in the literature to evaluate the effects of the COVID-19 pandemic on male fertility by comparing the demographic structure and spermiograms of patients who presented to a urology clinic due to infertility before and during the pandemic. The impact of the pandemic on semen parameters in men is a controversial issue and may occur in two ways. The first one is the effect of the infection itself, while the second one involves the possible psychophysiological effects of pandemic-imposed social restrictions on male reproductive health.

In the pathophysiology of COVID-19 infection, host ACE2 receptors facilitate intracellular entry and replication of the SARS-CoV-2 virus. This process is much easier in cells with high ACE2 expression. In many studies, the ACE2 expression level was found to be high in the seminiferous tubules and in Leydig 
and Sertoli cells. This potential affinity of the SARS-CoV-2 virus for the testicles is the main basis for the researchers who support this view. However, studies evaluating the effect of infection on semen parameters have yielded different results. In fact, it remains unclear whether the testicles and other male reproductive organs are susceptible to SARS-CoV-2 infection. Temiz et al. ${ }^{13}$ found that sperm quality decreased in the acute period of COVID-19 infection but did not differ from controls after treatment. They attributed the decrease in semen parameters during acute infection to high fever, but noted that SARS-CoV-2 was not detected by polymerase chain reaction (PCR) in the semen during this period ${ }^{13}$. In another study, SARS-CoV-2 was detected by PCR in the respiratory tract but not testicular tissue in postmortem evaluations after COVID-related deaths ${ }^{14}$.

The levels of sex hormones in individuals infected with COVID-19 have also been investigated due to their direct effect on semen parameters. Male sex hormones vary dramatically with acute illness or physiological stress, so it is important to remember that these early results may be disputable ${ }^{15}$. In a study of 119 patients, Ma et al. determined that luteinizing hormone and prolactin levels were higher in COVID-19 patients compared with the control group but did not detect a significant difference in testosterone or estradiol levels ${ }^{7}$. In another study, pretreatment levels of follicle-stimulating hormone, luteinizing hormone, and testosterone levels were lower in 30 COVID-19 patients compared with the control group, while posttreatment levels did not differ significantly from those of controls ${ }^{13}$. In a study of 31 patients, Rastrelli et al. ${ }^{16}$ reported that testosterone levels were significantly lower in COVID-19 patients who required intensive care than in those with mild disease ${ }^{16}$. It seems that the conversation on the effect of COVID-19 infection on testicular functions cannot be closed yet.

Another important aspect of the COVID-19 pandemic is the major lifestyle changes that have occurred for many individuals.
Social isolation during pandemic increased the feeling of loneliness and the usage of mobile devices, laptops, and computers. These devices may adversely affect semen parameters due to the low-level exposure of radio-frequency electromagnetic fields they produce ${ }^{17}$. In addition, obesity that can occur due to a sedentary lifestyle may also be an important risk factor. A meta-analysis study showed that semen quality was lower in obese men than in men with normal weight ${ }^{18}$. Psychosocial stress caused by the pandemic also constitutes a significant public health problem. Actually, the effect of psychosocial stress on semen parameters has been a topic of debate for a long time. In a prospective study, Hjollund et al. ${ }^{19}$ determined that there was no relationship between stress and sperm parameters ${ }^{19}$, whereas Janevic et al. ${ }^{20}$ found a negative association between stress and sperm concentration, motility, and morphology ${ }^{20}$. As the duration and degree of stress experienced by a lonely man cannot be measured with objective parameters, the effect of stress on semen parameters remains uncertain. The results of the present study suggest that these pandemic-related risk factors are not severe enough to disrupt sperm parameters. Of course, these 1-year results can also be seen as a preliminary study. If the pandemic continues, future studies of larger series will provide more insight into this issue.

It is also necessary to question the sex life of patients who present due to male infertility during the COVID-19 pandemic. Analyzing the semen parameters alone may not be enough in these patients. It has been observed in pandemics that patients affected by mental disorders far outnumber the infected patients ${ }^{21}$. Therefore, it is important to remember that increased anxiety or depression in men during the pandemic can also cause erectile dysfunction (ED) and loss of libido ${ }^{22}$. An individual's psychological state may trigger or exacerbate ED. Depression has been found to double the risk of $\mathrm{ED}^{23}$. $\mathrm{ED}$ may in turn cause a loss of self-esteem, which can adversely affect

Table 1. Spermiogram results by year.

\begin{tabular}{|c|c|c|c|c|}
\hline & Prepandemic group 1 (2018) & Prepandemic group 2 (2019) & Pandemic group (2020) & p-value \\
\hline Number of patients & 207 & 190 & 197 & 0.691 \\
\hline Age (years), mean $\pm S D$ & $36.61 \pm 7.21$ & $35.58 \pm 7.15$ & $33.15 \pm 6.36$ & $<0.001$ \\
\hline Semen volume $(\mathrm{mL})$, mean \pm SD & $3.96 \pm 1.74$ & $4.09 \pm 1.93$ & $4.09 \pm 1.78$ & 0.910 \\
\hline Normospermia rate $(\%, \mathrm{n})$ & $29(60)$ & $27.4(52)$ & $35(69)$ & \\
\hline Oligoasthenoteratoozospermia $(\%, n)$ & $24.2(50)$ & $25.8(49)$ & $17.3(34)$ & \\
\hline Asthenoteratoozospermia $(\%, \mathrm{n})$ & $16.9(35)$ & $17.4(33)$ & $18.8(37)$ & \\
\hline Asthenoozospermia $(\%, n)$ & $11.6(24)$ & $13.7(26)$ & $16.8(33)$ & \\
\hline Teratozoospermia $(\%, n)$ & $10.1(21)$ & $7.4(14)$ & $6.6(13)$ & \\
\hline Cryptozoospermia (\%, n) & $2.4(5)$ & $3.2(6)$ & $1.5(3)$ & \\
\hline Azoospermia $(\%, n)$ & $5.8(12)$ & $5.3(10)$ & $4.1(8)$ & \\
\hline
\end{tabular}


their partner relationship. Therefore, performing a psychological evaluation is recommended before seeking an organic cause for $\mathrm{ED}$, especially in men under the age of $40^{24}$.

Another important issue during the pandemic is loss of libido9. Although this condition (also known as hypoactive sexual desire disorder) is less common in men than women, it can lead to important problems between couples ${ }^{25}$. In a prevalence study by Carvalheira et al. ${ }^{26}$, loss of libido lasting more than 2 months was most commonly seen among men aged 30-39 years ${ }^{26}$. Clinicians should bear in mind that low libido is not only caused by low testosterone levels, but can also occur as a side effect of antidepressant and antipsychotic drugs ${ }^{27}$. Furthermore, sexual performance anxiety, relationship problems between couples, and depression/anxiety reduce sexual desire ${ }^{28}$.

One of the important findings of our study was that the mean age of men presenting due to infertility during the pandemic was $33.1 \pm 6.3$ years, significantly younger than in the previous 2 years. This difference may reflect an increase in future anxiety in these patients during the first year of the pandemic. A study in the literature demonstrated that the prevalence of anxiety/depression during the COVID-19 pandemic increased significantly more among participants $<35$ years of age than in participants $>35$ years of age ${ }^{15}$. According to the definition of infertility, pregnancy is not achieved for 12 months despite timed or regular sexual intercourse. Therefore, a detailed history should be obtained from infertile couples to understand the frequency of sexual intercourse during the COVID-19 pandemic. The lack of difference in the number of patients presenting to the clinic due to infertility compared with the previous 2 years indicates that there was no change in couples' desire to have children, despite the adverse circumstances brought about by the COVID-19 pandemic.

\section{CONCLUSION}

The available data suggest that for patients who present due to infertility during the pandemic and have no major problems

\section{REFERENCES}

1. Zandifar A, Badrfam R. Iranian mental health during the COVID-19 epidemic. Asian J Psychiatr. 2020;51:101990. https://doi. org/10.1016/j.ajp.2020.101990

2. Franchim CS, Soares-Junior JM, Serafini PC, Monteleone PAA, Coccuzza MS, Zanardo EA, et al. Efficacy of MLPA for detection of Y-chromosome microdeletions in infertile Brazilian patients. J Assist Reprod Genet. 2020;37(5):1251-9.https://doi.org/10.1007/ s10815-020-01777-8

3. DeMartinH,CocuzzaMS,TiseoBC,WoodGJA, MirandaEP,Monteleone PAA, et al. Positive rheotaxis extended drop: a one-step procedure to select and recover sperm with mature chromatin for intracytoplasmic with semen parameters, a thorough history should be obtained, and psychiatric evaluation is performed to question about sexual desire and presence of ED.

The limitation of this study is the lack of data regarding whether the patients who presented during the pandemic had a history of COVID-19 infection. However, the main objective of this study was not to evaluate the effect of COVID-19 infection specifically but to demonstrate the overall impact of the pandemic. Therefore, this study did not examine the history of COVID-19 infection in the patients who presented due to infertility during the pandemic. Moreover, the results of this study pertain to the first year of the pandemic. As the pandemic continues, the results of future long-term studies will be more valuable. In addition, specific sperm changes were not evaluated and dynamic analysis was not performed in this study ${ }^{2,3}$.

During the first year of the COVID-19 pandemic, there was no significant difference in the number of men who presented to the urology outpatient clinic due to infertility or in the patients' pathological results in terms of semen volume and sperm count, motility, and morphology when compared with data from 2018 and 2019. However, the men who presented for treatment during the pandemic were significantly younger than those in the previous 2 years. Questioning about psychosexual behavior may also be considered when evaluating infertile men during the pandemic.

\section{ETHICAL APPROVAL}

The study protocol was approved by the local ethics committees.

\section{AUTHORS' CONTRIBUTIONS}

MS: Conceptualization, Methodology, Writing - original draft.

MD: Data curation, Resources, Writing - review \& editing. ME: Formal Analysis, Software. EYK: Funding acquisition. SSU: Investigation, Validation, Visualization. AS: Project administration. HT: Supervision.

sperm injection. J Assist Reprod Genet. 2017;34(12):1699-708. https://doi.org/10.1007/s10815-017-1024-1

4. Vander Borght M, Wyns C. Fertility and infertility: definition and epidemiology. Clin Biochem. 2018;62:2-10. https://doi. org/10.1016/j.clinbiochem.2018.03.012

5. Barton LM, Duval EJ, Stroberg E, Ghosh S, Mukhopadhyay S. COVID-19 autopsies, Oklahoma, USA. Am J Clin Pathol. 2020;153(6):725-33. https://doi.org/10.1093/ajcp/aqaa062

6. Pan F, Xiao X, Guo J, Song Y, Li H, Patel DP, et al. No evidence of severe acute respiratory syndrome-coronavirus 2 in semen of males recovering from coronavirus disease 2019. Fertil Steril. 2020;113(6):1135-9. https://doi.org/10.1016/j. fertnstert.2020.04.024 
7. Ma L, Xie W, Li D, Shi L, Ye G, Mao Y, et al. Evaluation of sex-related hormones and semen characteristics in reproductive-aged male COVID-19 patients. J Med Virol. 2021;93(1):456-62. https://doi. org/10.1002/jmv.26259

8. Guo L, Zhao S, Li W, et al. Absence of SARS-CoV-2 in semen of a COVID-19 patient cohort. Andrology. 2021;9(1):42-7. https://doi. org/10.1111/andr.12848

9. Ibarra FP, Mehrad M, Mauro MD, Godoy MFP, Cruz EG, Nilforoushzadeh MA, et al. Impact of the COVID-19 pandemic on the sexual behavior of the population. The vision of the east and the west. Int Braz J Urol. 2020;46(Suppl 1):104-12. https:// doi.org/10.1590/S1677-5538.IBJU.2020.S116

10. Lehmiller JJ, Garcia JR, Gesselman AN, Mark KP. Less sex, but more sexual diversity: Changes in sexual behavior during the COVID-19 coronavirus pandemic. Leis Sci. 2020;43(1-2):295-304. https:// doi.org/10.1080/01490400.2020.1774016

11. Facio Junior FN, Facio MFW, Spessoto ACN, Spessoto LCF. Sexual behavior in men during COVID-19. Rev Assoc Med Bras. 2020;66(12):1613-4. https://doi.org/10.1590/18069282.66.12.1613

12. Esteves SC. Clinical relevance of routine semen analysis and controversies surrounding the 2010 World Health Organization criteria for semen examination. Int Braz J Urol. 2014;40(4):44353. https://doi.org/10.1590/S1677-5538.IBJU.2014.04.02

13. Temiz MZ, Dincer MM, Hacibey I, Yazar RO, Celik C, Kucuk SH, et al. Investigation of SARS-CoV-2 in semen samples and the effects of COVID-19 on male sexual health by using semen analysis and serum male hormone profile: a cross-sectional, pilot study. Andrologia. 2020;53(2):e13912. https://doi.org/10.1111/and.13912

14. Flaifel A, Guzzetta M, Occidental M, Najari BB, Melamed J, Thomas $\mathrm{KM}$, etal. Testicular changes associated with severe acute respiratory syndrome coronavirus 2 (SARS-CoV-2). Arch Pathol Lab Med. 2021;145(1):8-9. https://doi.org/10.5858/arpa.2020-0487-LE

15. Huang Y,Zhao N. Generalized anxiety disorder, depressive symptoms and sleep quality during COVID-19 outbreak in China: a web-based cross-sectional survey. Psychiatry Res. 2020;288:112954. https:// doi.org/10.1016/j.psychres.2020.112954

16. Rastrelli G, Di Stasi V, Inglese F, Beccaria M, Garuti M, Di Costanzo $D$, et al. Low testosterone levels predict clinical adverse outcomes in SARS-CoV-2 pneumonia patients. Andrology. 2021;9(1):88-98. https://doi.org/10.1111/andr.12821
17. Balawender K, Orkisz S. The impact of selected modifiable lifestyle factors on male fertility in the modern world. Cent Eur J Urol. 2020;73(4):563-568. https://doi.org/10.5173/ceju.2020.1975

18. Halles C, Carroll M, Fryar C, Ogden C. Prevalence of obesity among adults and youth: United States, 2015-2016. NCHS Data Brief. 2017;(288):1-8. PMID: 29155689

19. Hjollund NHI, Bonde JPE, Henriksen TB, Giwercman A, Olsen J, Danish First Pregnancy Planner Study Team. Reproductive effects of male psychologic stress. Epidemiology. 2004;15(1):21-7. https:// doi.org/10.1097/01.ede.0000100289.82156.8b

20. Janevic T, Kahn LG, Landsbergis P, Cirillo PM, Cohn BA, Liu X, et al. Effects of work and life stress on semen quality. Fertil Steril. 2014;102(2):530-8.https://doi.org/10.1016/j.fertnstert.2014.04.021

21. Ekmekci EI. COVID-19 pandemic and community mental health. In: Coşar B, editor. Psychiatry and Covid-19. 1st ed. Ankara: Türkiye Klinikleri; 2020. p. 12-7.

22. Duran MB, Yildirim O, Kizilkan Y, Tosun C, Cirakoglu A, Gultekin MH etal. Variations in the number of patients presenting with andrological problems during the coronavirus disease 2019 pandemic and the possible reasons for these variations: a multicenter study. Sex Med. 2021;9(1):100292. https://doi.org/10.1016/j.esxm.2020.100292

23. Benet AE, Melman A. The epidemiology of erectile dysfunction. Urol Clin North Am. 1995;22(4):699-709. PMID: 7483123

24. Yafi FA, Jenkins L, Albersen M, Corona G, Isidori AM, Goldfarb S, et al. Erectile dysfunction. Nat Rev Dis Prim. 2016;2:16003. https://doi.org/10.1038/nrdp.2016.3

25. Revicki DA, Althof SE, Derogatis LR, Kingsberg SA, Wilson H, Sadiqet A, al. Reliability and validity of the elements of desire questionnaire in premenopausal women with hypoactive sexual desire disorder. J Patient Rep Outcomes. 2020;4(1): 82. https:// doi.org/10.1186/s41687-020-00241-6

26. Carvalheira A, Traeen B, Štulhofer A. Correlates of men's sexual interest: a cross-cultural study. J Sex Med. 2014;11(1):154-64 https://doi.org/10.1111/jsm.12345

27. Knegtering H, Bruggeman R, Castelein S, Wiersma D. Antipsychotics and sexual functioning in persons with psychoses. Tijdschr Psychiatr. 2007;49(10):733-42. PMID: 17929226

28. Corona G, Mannucci E, Schulman C, Petrone L, Mansani R, Cilotti A, et al. Psychobiologic correlates of the metabolic syndrome and associated sexual dysfunction. Eur Urol. 2006;50(3):595-604 https://doi.org/10.1016/j.eururo.2006.02.053 